Monatsschrift f. Geburtshülfe u. Gynäkologie 1933;93:I-IV

\title{
Contents, Vol. 93, 1933
}

Inhaltsverzeichnis.

Oríginalarbeiíen. $\quad \mathrm{s} \beta \mathrm{u} \beta$

Biró, Stefan, Gruppenspezifische Eigenschaften der Frauenmilch und

des Kolostrums $\quad 354$

Cahn, Nicolai, 1st das Zurückbleiben eines Fremdkörpers in der Bauch-höhle als fabrlässige

Körperverletzung strafbar? Aus einem Ober-gutachten. Bemerkung zum Auí'satz von A.

Döderlein, München

(diese Monatsscbrift Bd. 91, Jahrgang 1932) 374

Döderlein, A., siehe Cabn, Nicolai.

Eberhart, F., Über den Wert der Excisio mueosae uteri 369

Ebrbardt, K., Über Coraminweckwirkung bei Pernoktonnarkose . . 335 Eufinger, Heinrich, Die

Beeinflussung des Giykogenblutspiegels

durch die Gestation und den mensuellen Zyklus $\quad 51$

-, M. ßothermundt und H. Wiesbader, Die trypanozide Eigen-

schaft des Serums in ihrer Bedeutung als Leberfunktionsprüfung

während der Gestationsperiode und beim Uteruskarzinom 249

Geller, Fr. Chr., Pränatale Ernährungs- und Belichtungseinflüsse . . 359

Guthmann, Heinrich, und Fritz Stähler, Die Beeinflussung der

Lage und Funktion des Verdauungskanals durch die normale

Schwangerschaft $\quad 327$

$\mathrm{H}$ a a s e, W., Blutgruppenuntersuchungen bei Eklamptischen, ihren Kindern

und deren Vätern 262

Habbe, Karl, Über das Vorkommen der Geburt in hinterer Hinter-

hauptslage in dem Material der Göttinger Frauenklinik der letzten

fünf Jahre $\quad 258$

Hofmann, Herbert, Ein Fall von Spontanheilung eines Chorion-

epithelioms der Scheide $\quad 311$

Kochmann, Gotthard, Erfahrungen mit der Bluttransfusion bei der puerperalen Sepsis und bei der sekundären Anämie 154

Köhler, Alfred, Beitrag zur Kenntnis der angeborenen Hautveränderungen 265

Kolegajew, G. A., Zur Frage über die Ätiologie und die bösartige

Degeneration der Leukopll·lkien der Portioschleimhaut 166

Kriwsky, L. A., Die Extrauterinschwangerschaft nach den Materialien

des Njecajew-Krankenhauses (ehemaliges Obuchow-Krankenhaus) zu

Leningrad 292

Kulka, Eugen, Über Hypophysenhinterlappenhormon im Liquor

cerebrospinalis und in der Milch 348

Luh, Beinhard, Zur Frage der isthmico-zervikalen Eieinbettung . . 277 
Martin, Ed., § $218 \quad 14$

Noetzel, Theodor, Der Zweckgedanke im $\S 218$ StGB 1

Riebold, Georg. Die Beeinflussung körperlicher Perioden durch

kosmische Wellen 163

EothermuDd, M., siehe Eufinger, H.

Schneider, G. H., Die amenorrhoischen Symptomkomplexe 83

Spiegler, R., Die Bedeutung der Zustandsformen des Kalkes und ihre

Beeinflussung durch das Parathyreoidea-Hormon 60

Stähler, Fritz, siehe Guthmann, Heinrich.

Stolte, K., Wechselseitige Beeinflussung von Mutter und Fötus . . 340

$1 Y$ Inhaltsverzeichnis.

Seit $\beta$ Syrowatko, Th, Zur Frage der konservativen Therapie von Uterusperíbrationen bei künstlichen Aborten

Tausch, M., Beitrag zur Frage der abnorm langen Schwangerschaftsdauer 137

Terechowa, A., Zur Frage über die morphologischen und funktionelien Beziehungen zwischen den Fötalelementen und der Uterustubenwand während der Gravidität $\quad 66$

Toll as, Helmut, Medikamentöse Geburtsbeschleunigung durch Solvochio auf parenteralem, peroralem und rektalem Wege 144

Traube, Karl, Klinische Erfahruvgen mit kombinierten und fraktionierten, wehenerregenden Hypophysenhinterlappenextrakten (Thymophysin, Orasthin) 301

Unseld, E., Über Vulvovagínitis diphtherica bei einem achtjährigen

Mädchen 177

Wiesbader, H., siehe Eufinger, $\mathrm{H}$.

Willi, Cas. , Unsere Erfahrungen mit Thymophysin 42

LudwigPiskacek $\dagger \quad 134$

D. Pulvermacher $\dagger \quad 136$

Grenzgebíete der Geburtshilfe und Gynäkologie.

Die Röntgentherapie in der Gynäkologie vom Juli 1931 bis Juli 1932.

Von Franz Wittenbeck, Erlangen 106

Bericht über innersekretorische Literatur (November 1931 bis Oktober 1932). Von Privatdozent Dr. K. Ehrhardt und Dr. E. Bruchhorst,

Frankfurt a. M. . , 181

Serologie des Karzinoms und der Schwangerschaft. Von Dr. Erich

Tscheme, Graz 314

Cholezystitis, Cholelithiasis, Appendizitis. Von Dr. HerbertHofmann,

Prag .377

Narkose und Dämmerschlaf. Übersichtsreferat über die Literatur des

Jahres 1932. Von Dr. C. Schroeder, Würzburg 384

Sitzungsberichte aus geburtshilflích-gynäkologischen Ges\&llschaften.

Gynäkologische Gesellscliaft zu Breslau. Sitzung vom 29. November 1932395

Niederrheinisch-westiälische Gesellschaft für Gynäkologie und Geburts

hilfe. 97. Sitzung vom 16. Januar 1932 zu Düsseldorf

124

Bayerische Gesellschaft für Geburtshilfe und Frauenheilkunde. Sitzung 
vom 7. Februar 1932 zu München 196

Geburtshilflich-gynäkologische Gesellschaft in Wien. Sitzung vom 8. No vember 1932240

Zweiter Internationaler Lichtkongreß in Kopenhagen vom 14. bis 18. August 1932242

Buchbesprechungen 243, 402

Perso $\pi$ alien und Tagesnachrichten $\quad 136,248,326,402$ 